\title{
Breeding for high sugar content, plant stalk juice and plant height characters in sweet sorghum
}

\author{
Uzun B.*, Guden B. \\ Department of Field Crops, Faculty of Agriculture, Akdeniz University, Antalya, Turkey \\ *e-mail: bulentuzun@akdeniz.edu.tr
}

Sweet sorghum (Sorghum bicolor L. Moench) with high biomass and high sugar content in its stalk is one of the most important energy crops. The high correlation between plant height and biomass and fermentable sugar in stem make sweet sorghum an important plant as a renewable bioenergy source. The potential of higher biomass and bioethanol with lower input in sweet sorghum comparing to maize make this crop valuable in large areas worldwide. The important characters for obtaining high biomass and sugar are mainly sugar and stem juice content per plant and plant height. 551 accessions were screened and several superior accessions were determined for these bioenergy related characters. Pyramiding different QTLs in a single line would obviously result in higher plant height, sugar content and biomass as the characters studied were under additive genetic behavior. QTL mapping is crucial to find out different QTLs for each character and thus "Erdurmus" line with high sugar content, plant height and stem juice was crossed with Ogretmenoglu which has contrasting values for the characters. The $F_{1} s$ were selfed and the segregating $\mathrm{F}_{2}$ population were generated. $376 \mathrm{~F}_{2}$ individuals were phenotyped for the bioenergy related characters. Of those, 200 individual DNAs were extracted and subjected to genotyping by sequencing (GBS) analyses. The corresponding $\mathrm{F}_{3}$ lines will be phenotyped in two different environments in this summer. PCR-based molecular markers developed from the SNP regions related to the characters will be tested in other advance lines which have high sugar content, plant height and stem juice. If the marker produces no information about any advance line, it will be assumed that this line may contain different QTL(s) and it will be used for pyramiding. This study proposes an authentic pipeline to find out different QTLs.

Acknowledgements: This study was supported by TUBITAK with a project number of 1180169. 